Journal for ImmunoTherapy of Cancer

\section{Phase II, single-arm trial of preoperative short-course radiotherapy followed by chemotherapy and camrelizumab in locally advanced rectal cancer}

To cite: Lin Z, Cai M, Zhang $\mathrm{P}$, et al. Phase II, singlearm trial of preoperative short-course radiotherapy followed by chemotherapy and camrelizumab in locally advanced rectal cancer. Journal for ImmunoTherapy of Cancer 2021;9:e003554. doi:10.1136/ jitc-2021-003554

- Additional supplemental material is published online only. To view, please visit the journal online (http://dx.doi.org/10. 1136/jitc-2021-003554).

ZL, MC and PZ contributed equally.

Accepted 01 October 2021

Check for updates

(c) Author(s) (or their employer(s)) 2021. Re-use permitted under CC BY-NC. No commercial re-use. See rights and permissions. Published by BMJ.

For numbered affiliations see end of article.

\section{Correspondence to}

Professor Tao Zhang; taozhangxh@hust.edu.cn

Professor Kaixiong Tao; kaixiongtao@hust.edu.cn

\section{ABSTRACT}

Background In locally advanced rectal cancer (LARC), preoperative short-course radiotherapy (SCRT) with delayed surgery has been shown to be as effective as long-course chemoradiotherapy, with only modest benefits. This study aimed to evaluate the efficacy and safety of preoperative SCRT combined with subsequent CAPOX (capecitabine and oxaliplatin) and the anti-PD-1 antibody camrelizumab in patients with LARC.

Methods This was a prospective, single-arm, phase II trial. Treatment-naïve patients with histologically confirmed T3-4N0M0 or T1-4N+M0 rectal adenocarcinoma received $5 \times 5$ Gy SCRT with two subsequent 21-day cycles of CAPOX plus camrelizumab after 1 week, followed by radical surgery after 1 week. The primary endpoint was pathological complete response ( $\mathrm{pCR}$ ) rate. Biomarker analysis was performed to identify a potential predictor of pCR to treatment.

Results From November 7, 2019 to September 14, 2020, 30 patients were enrolled, and 27 patients received at least one dose of CAPOX plus camrelizumab. Surgery was performed in 27 (100\%) patients. The pCR (ypTON0) rate was $48.1 \%$ (13/27), including 46.2\% (12/26) for proficient mismatch repair (MMR) tumors and 100\% (1/1) for deficient MMR tumors. Immune-related adverse events were all grade 1-2, with the most common being reactive cutaneous capillary endothelial proliferation (81.5\%). No grade $4 / 5$ adverse events occurred. Biomarker analysis showed patients without FGFR1-3 deletions had a better tendency for pCR.

Conclusions SCRT combined with subsequent CAPOX plus camrelizumab followed by delayed surgery showed a favorable $\mathrm{pCR}$ rate with good tolerance in patients with LARC, especially in the proficient MMR setting. A randomized controlled trial is ongoing to confirm these results.

Trial registration number ClinicalTrials.gov identifier: NCT04231552.

\section{INTRODUCTION}

Rectal cancer, which accounts for more than one-third of colorectal cancer (CRC) cases, ${ }^{1}$ has been challenging in terms of treatment and organ preservation, especially for locally advanced rectal cancer (LARC), because of the complex anatomical structures and high rates of postoperative complications and local recurrence. Currently, multidisciplinary therapy based on neoadjuvant long-course chemoradiotherapy (LC-CRT) with delayed surgery or short-course radiotherapy (SCRT) with immediate surgery is the predominant treatment strategy for LARC. ${ }^{2}$ The Stockholm III trial of patients with LARC was the first to demonstrate that SCRT with delayed surgery was superior to SCRT with immediate surgery and long-course radiotherapy (LCRT) with delayed surgery in terms of pathological complete response (pCR) and tumor downstaging. ${ }^{34}$ Recently, a novel treatment combination for LARC patients, namely, neoadjuvant short-course hypofractionated radiotherapy combined with subsequent chemotherapy followed by delayed surgery, led to a greater pCR and had a lower 3-year disease-related treatment failure rate than LC-CRT followed by delayed surgery. ${ }^{5}$ However, unfortunately, the pCR rate is consistently below $30 \%$, which is not good in terms of meeting treatment needs or enabling organ preservation for patients with LARC; therefore, there is a need to explore new neoadjuvant treatment options.

In 2015, immunotherapy was shown to provide significant clinical benefits to patients with metastatic CRC with a deficient mismatch repair (dMMR)/microsatellite instability-high (MSI-H) status, whereas no response was observed in patients with a proficient mismatch repair (pMMR)/ microsatellite stable (MSS) status, which accounts for approximately 96\%-98\% of metastatic CRCs and $85 \%$ of all CRCs. ${ }^{7-9}$ Of 
note, compared with patients with advanced-stage CRC, early-stage patients have higher $\mathrm{CD}^{+} \mathrm{T}$-cell infiltration, an increase that could enhance the response to immune checkpoint inhibitors, as previously reported in melanoma. ${ }^{1011}$ In addition, the preliminary data in the NICHE study demonstrated that $13 \%$ of patients with early-stage pMMR colon cancer could benefit from neoadjuvant dual immunotherapy, consistent with reports in other solid tumors that early-stage disease may be more responsive to immunotherapy, particularly as neoadjuvant therapy. ${ }^{12} 13$

Numerous studies have demonstrated that the combination of checkpoint blockade immunotherapy and radiotherapy could generate synergistic antitumor effects against local and distant tumors. ${ }^{14-16}$ Recently, the clinical efficacy and safety of preoperative LC-CRT and subsequent nivolumab monotherapy in the treatment of rectal cancer were described in the VOLTAGE-A study, which enrolled and assessed 37 patients with MSS LARC. ${ }^{17}{ }^{18}$ A pCR rate of $30 \%$ and a major pathologic response rate of $38 \%$ were observed, with tolerable toxicities. Given previous studies suggesting that short-course hypofractionated radiotherapy combined with subsequent chemotherapy is comparable with LC-CRT in terms of the effects of neoadjuvant therapy in rectal cancer and that the immune response was increased by hypofractionated radiotherapy plus programmed death 1 (PD-1) blockade, ${ }^{19}$ we hypothesized that neoadjuvant short-course hypofractionated radiotherapy combined with subsequent chemotherapy and immune checkpoint inhibitors followed by delayed surgery would afford clinical benefits for LARC.

We report the short-term results from a single-arm, single-center, phase II trial evaluating the efficacy and safety of preoperative short-course radiotherapy combined with subsequent chemotherapy (capecitabine and oxaliplatin) and the anti-PD-1 antibody camrelizumab in patients with LARC.

\section{METHODS}

\section{Study design and participants}

This was a prospective, single-arm, single-center, phase II trial performed at the Union Hospital affiliated to Tongji Medical College of Huazhong University of Science and Technology in China.

Eligible patients were aged 18 to 75 years, with histologically confirmed T3-4N0M0 or T1-4N+M0 rectal adenocarcinoma, inferior margin of $\leq 10 \mathrm{~cm}$ from the anal verge, treatment naïve, an Eastern Cooperative Oncology Group performance status of $0-1$, and no severe hematologic, cardiac, pulmonary, hepatic, or renal functional abnormalities or immunodeficiency diseases. Laboratory tests were required to meet show the following: hemoglobin level $\geq 9 \mathrm{~g} / \mathrm{dL}$; white blood cell count $\geq 3 \times 10^{9} / \mathrm{L}$; absolute neutrophil count $\geq 1.5 \times 10^{9} / \mathrm{L}$; platelet count $\geq 100 \times 10^{9}$ $/ \mathrm{L}$; bilirubin level $<1.5 \times$ the upper limit of normal (ULN); aspartate aminotransferase and alanine aminotransferase (AST) levels $\leq 2.5 \times$ ULN; serum creatinine level $\leq 1.5 \times$ ULN or creatinine clearance $\geq 50 \mathrm{~mL} / \mathrm{min}$; thyroid-stimulating hormone level $\leq 1 \times$ ULN or T3 and T4 levels within normal limits. Patients were recruited regardless of their programmed death ligand 1 (PD-L1) expression level. Key exclusion criteria included previous exposure to any anti-PD-1 or anti-PD-L1 antibody, a history of pelvic radiation, treatment with corticosteroids or other immunosuppressive agents within 14 days prior to study drug administration, presence of autoimmune disease, and known interstitial lung disease.

\section{Procedures}

Eligible patients received SCRT ( $5 \times 5$ Gy over 5 days), followed 1 week later by two subsequent 21-day cycles of CAPOX (oxaliplatin $130 \mathrm{mg} / \mathrm{m}^{2}$ intravenously, day 1; capecitabine $1000 \mathrm{mg} / \mathrm{m}^{2}$ oral twice daily, days 1-14) plus camrelizumab (200 mg intravenous drip, day 1), followed by radical surgery after 1 week. Surgery was done according to total mesorectal excision principles. Low anterior resection was performed for middle and low rectal cancers with distal margins of more than $1 \mathrm{~cm}$, and abdominoperineal resection was conducted by the surgeon for extremely low tumors.

Patients received a baseline assessment including collection of information on demographics, medical history, and disease characteristics before enrollment, and they underwent systematic physical examination and relevant laboratory and imaging (chest CT, liver MRI, abdominal and pelvic CT or MRI) tests before and after treatment. Patients discontinuing treatment for reasons other than progressive disease were followed up every 3 months until disease relapse or death.

Surgically resected specimens were processed and examined as previously reported. ${ }^{20}$ The extent of the residual tumor in the resected specimens was classified as per the 8th edition of the International Union Against Cancer TNM staging system. All resected specimens (including the whole tumor bed and surrounding tissues) were sampled and cross-sectioned consecutively. If no residual viable tumor was identified after examination, additional three-level sections were carried out. All sampled lymph nodes were examined based on standard procedures. All sections were evaluated independently by two senior pathologists. A pCR was defined as the absence of any remaining viable cancer cells in the resected primary tumor specimen and all sampled regional lymph nodes (ypT0N0). The tumor regression grade (TRG) was assessed according to the criteria proposed by Ryan $e t \mathrm{al}^{21}$

\section{Outcomes}

The primary endpoint was pCR rate, defined as the proportion of patients who had a pCR. Secondary endpoints included 3-year event-free survival rate (defined as the percentage of patients without disease recurrence or progression or death due to any cause at the 3-year follow-up), R0 resection rate (defined as the rate of negative margins microscopically), 3-year overall survival rate (defined as the percentage of patients alive at the 3-year follow-up), complication rate, safety, and 


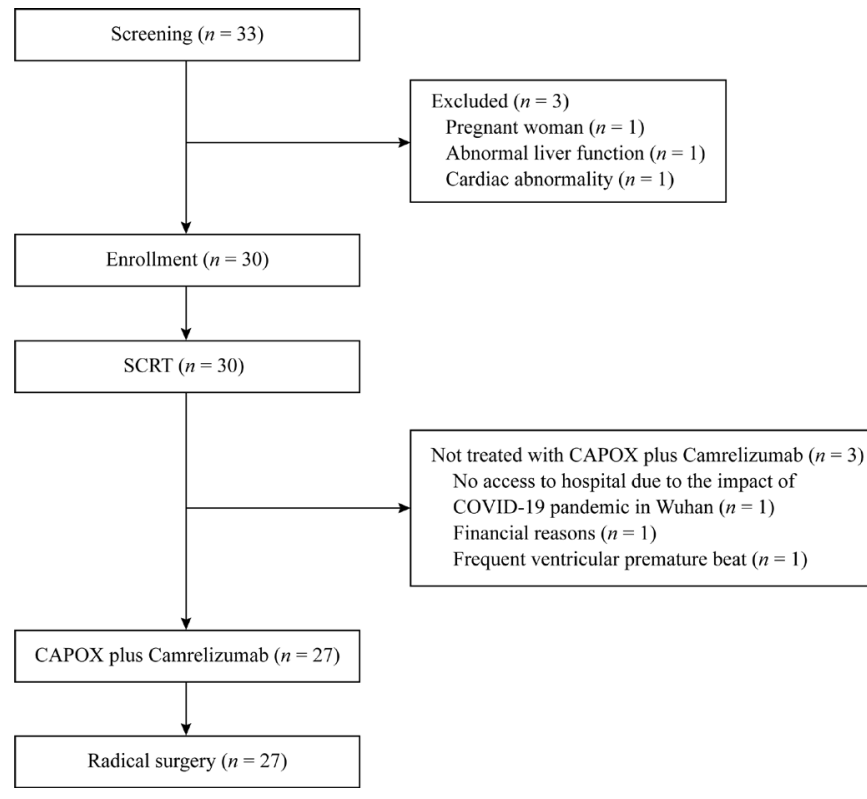

Figure 1 Trial profile. CAPOX, capecitabine plus oxaliplatin; SCRT, short-course radiotherapy.

quality of life. Adverse events (AEs), recorded during the period when patients signed their informed consent forms to 90 days after surgery, were graded according to the National Cancer Institute Common Terminology Criteria for Adverse Events, version 5.0.

\section{Biomarker analysis}

Tissue samples or paraffin sections were required to be provided when patients were enrolled. Immunohistochemistry was performed to detect the expression of PD-L1, DNA mismatch repair (MMR) proteins (MSH6, MSH2, MLH1, and PMS2), CD4, and CD8. The PD-L1 combined positive score (CPS) was evaluated using the PD-L1 IHC 22C3 pharmDx assay (Agilent Technologies, Santa Clara, CA, USA), defined as the number of PD-L1 positive cells (tumor cells, lymphocytes, macrophages) as a proportion of the total number of tumor cells multiplied by 100. Positive PD-L1 expression was considered when the CPS was 1 or more. Comprehensive genomic profiling was conducted using targeted gene capturebased next-generation sequencing technology. Briefly, for formalin-fixed paraffin-embedded tissues, H\&E staining was performed, and the stained sections were evaluated by a pathologist to ensure tumor cells $\geq 20 \%$. DNA was extracted from the tumor tissues of patients using standard methods. A panel of 418 genes was captured and then sequenced through the Illumina NovaSeq 6000 platform (Illumina, San Diego, CA, USA) with $2 \times 150 \mathrm{bp}$ paired-end reads. The average sequencing depth of tumor tissues was $\geq 1000 \times$. Genomic alterations, including tumor mutation burden (TMB), single nucleotide variants, short and long insertions and deletions, copy number variants, and gene fusions, were assessed.
Table 1 Patient characteristics

\begin{tabular}{|c|c|}
\hline Characteristics & Patients $(n=30)$ \\
\hline Age, years, median (range) & $57(31-73)$ \\
\hline \multicolumn{2}{|l|}{ Sex, n (\%) } \\
\hline Male & $17(56.7)$ \\
\hline Female & $13(43.3)$ \\
\hline \multicolumn{2}{|c|}{ ECOG performance status, $n(\%)$} \\
\hline 0 & $13(43.3)$ \\
\hline 1 & $17(56.7)$ \\
\hline \multicolumn{2}{|l|}{ Clinical T category, n (\%) } \\
\hline сT3 & $22(73.3)$ \\
\hline cT4 & $8(26.7)$ \\
\hline \multicolumn{2}{|l|}{ Clinical $\mathrm{N}$ category, $\mathrm{n}(\%)$} \\
\hline cNO & $4(13.3)$ \\
\hline $\mathrm{cN} 1$ & $16(53.3)$ \\
\hline $\mathrm{cN} 2$ & $10(33.3)$ \\
\hline \multicolumn{2}{|l|}{ Clinical disease stage, $\mathrm{n}(\%)$} \\
\hline Stage II (cT3-4N0) & $4(13.3)$ \\
\hline Stage III (cT1-4N1-2) & $26(86.7)$ \\
\hline \multicolumn{2}{|l|}{ CRM, n (\%) } \\
\hline Positive & $21(70.0)$ \\
\hline Negative & $9(30.0)$ \\
\hline \multicolumn{2}{|l|}{ EMVI, n (\%) } \\
\hline Positive & $12(40.0)$ \\
\hline Negative & $18(60.0)$ \\
\hline \multicolumn{2}{|c|}{ Distance from primary tumor to anal verge, $\mathrm{n}(\%)$} \\
\hline Median (range), cm & $4.7(1.9-9.0)$ \\
\hline$<5$ & $15(50.0)$ \\
\hline $5-10$ & $15(50.0)$ \\
\hline \multicolumn{2}{|l|}{ Length of tumor lesion, $\mathrm{cm}$} \\
\hline Mean (SD) & $5.5(1.7)$ \\
\hline Median (range) & $5.4(2.1-10.0)$ \\
\hline \multicolumn{2}{|l|}{ Mismatch repair status, $\mathrm{n}(\%)$} \\
\hline dMMR & $1(3.3)$ \\
\hline pMMR & 28 (93.3) \\
\hline Unknown & $1(3.3)$ \\
\hline \multicolumn{2}{|l|}{ PD-L1 expression, CPS, n (\%) } \\
\hline Negative & $20(66.7)$ \\
\hline Positive ( $\geq 1)$ & $6(20.0)$ \\
\hline Unknown & $4(13.3)$ \\
\hline \multicolumn{2}{|c|}{ Tissue-based TMB (mut/Mb), n (\%) } \\
\hline$<10$ & $7(23.3)$ \\
\hline$\geq 10$ & $7(23.3)$ \\
\hline Unknown & $16(53.3)$ \\
\hline \multicolumn{2}{|l|}{ Baseline CEA level, n (\%) } \\
\hline Normal (<5 ng/mL) & $17(56.7)$ \\
\hline Abnormal ( $\geq 5 \mathrm{ng} / \mathrm{mL}$ ) & $13(43.3)$ \\
\hline
\end{tabular}

CEA, carcinoembryonic antigen; CPS, combined positive score; CRM, circumferential resection margin; dMMR, deficient mismatch repair; ECOG, Eastern Cooperative Oncology Group; EMVI, extramural vascular invasion; PD-L1, programmed death ligand 1; pMMR, proficient mismatch repair; TMB, tumor mutation burden. 
Table 2 Postoperative pathological response

\begin{tabular}{|c|c|c|c|}
\hline & Total $(n=27)$ & pMMR $(n=26)$ & dMMR $(n=1)$ \\
\hline pCR (ypTONO), n (\%) & $13(48.1)$ & $12(46.2)$ & $1(100.0)$ \\
\hline \multicolumn{4}{|l|}{ T category, n (\%) } \\
\hline ypTis & $1(3.7)$ & $1(3.8)$ & 0 \\
\hline урт2 & 5 (18.5) & $5(19.2)$ & 0 \\
\hline \multicolumn{4}{|l|}{$\mathrm{N}$ category, $\mathrm{n}(\%)$} \\
\hline ypNO & $19(70.4)$ & $18(69.2)$ & $1(100.0)$ \\
\hline ypN1 & $6(22.2)$ & $6(23.1)$ & 0 \\
\hline ypN2 & $2(7.4)$ & $2(7.7)$ & 0 \\
\hline \multicolumn{4}{|c|}{ Pathological stage, n (\%) } \\
\hline II & $5(18.5)$ & 5 (19.2) & 0 \\
\hline III & $8(29.6)$ & $8(30.8)$ & 0 \\
\hline \multicolumn{4}{|c|}{ Tumor regression grade, $\mathrm{n}(\%)$} \\
\hline 0 & $13(48.1)$ & $12(46.2)$ & $1(100.0)$ \\
\hline 1 & $5(18.5)$ & $5(19.2)$ & 0 \\
\hline 2 & $7(25.9)$ & $7(26.9)$ & 0 \\
\hline 3 & $2(7.4)$ & $2(7.7)$ & 0 \\
\hline
\end{tabular}

dMMR, deficient mismatch repair; pMMR, proficient mismatch repair.

\section{Statistical analysis}

According to previous studies, the pCR rate after preoperative CRT in patients with LARC is approximately $15 \% .^{22} 23$ We expected that the regimen of SCRT combined with subsequent chemotherapy and camrelizumab could increase the pCR rate from $15 \%$ to $40 \%$. A sample size of 24 patients was required to provide at least $80 \%$ power to detect this estimated improvement in a one-sided $\chi^{2}$ test with a significance level of $2.5 \%$, and a $20 \%$ dropout was estimated, resulting in a total sample size of 30 patients planned for this study.

Statistical analyses were conducted using SAS software (V.9.2; SAS Institute, Cary, NC, USA). Continuous variables were summarized using medians and ranges, and categorical variables were described using frequencies and percentages. Baseline and safety analyses were performed for all enrolled patients (intention-to-treat (ITT) population), and efficacy analyses were conducted for those who were administered at least one dose of camrelizumab (full analysis set). Comparisons between the groups were performed using Fisher's exact test or the $\chi^{2}$ test. P values $<0.05$ were considered statistically significant.

\section{RESULTS}

\section{Patient characteristics and compliance}

From November 7, 2019 to September 14, 2020, the target number of eligible patients was enrolled $(n=30)$. Three patients were not treated with CAPOX plus camrelizumab for the reasons given in figure 1 and were excluded from the efficacy analysis. Patient baseline and disease characteristics are summarized in table 1. Approximately half of the patients had at least one high-risk factor, including cT4 disease in $26.7 \%(8 / 30)$, cN2 disease in $33.3 \%$ $(10 / 30)$, extramural vascular invasion in $40.0 \%(12 / 30)$, and tumors located within $<5 \mathrm{~cm}$ from the anal verge in $50.0 \%(15 / 30)$.

During the treatment period, all patients received the full irradiation dose of 25 Gy $(100 \%, 30 / 30)$, and 27 patients $(90.0 \%, 27 / 30)$ received at least one cycle of CAPOX plus camrelizumab, of whom three patients did not complete the second cycle of CAPOX plus camrelizumab as specified in the protocol due to the impact of the COVID-19 pandemic. The median intervals from the completion of preoperative SCRT to receiving subsequent CAPOX plus camrelizumab and from the last dose of CAPOX plus camrelizumab to surgery were 12 (range, 4-18) days and 14 (range, 5-141) days, respectively.

\section{Surgery and pathology}

A total of 27 patients $(100 \%, 27 / 27)$ underwent surgery in the full analysis set, with an $\mathrm{R} 0$ resection rate of $100 \%$ $(27 / 27)$ and anal preservation rate of $88.9 \%$ (24/27). A total of four patients developed postoperative complications, including infection in three patients $(11.1 \%, 3 / 27)$ and bleeding in one patient $(3.7 \%, 1 / 27)$. No other 
A

Pre-treatment
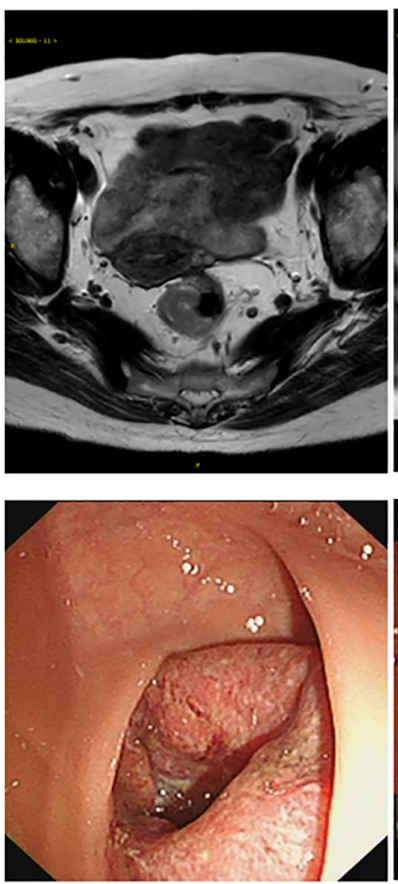

B

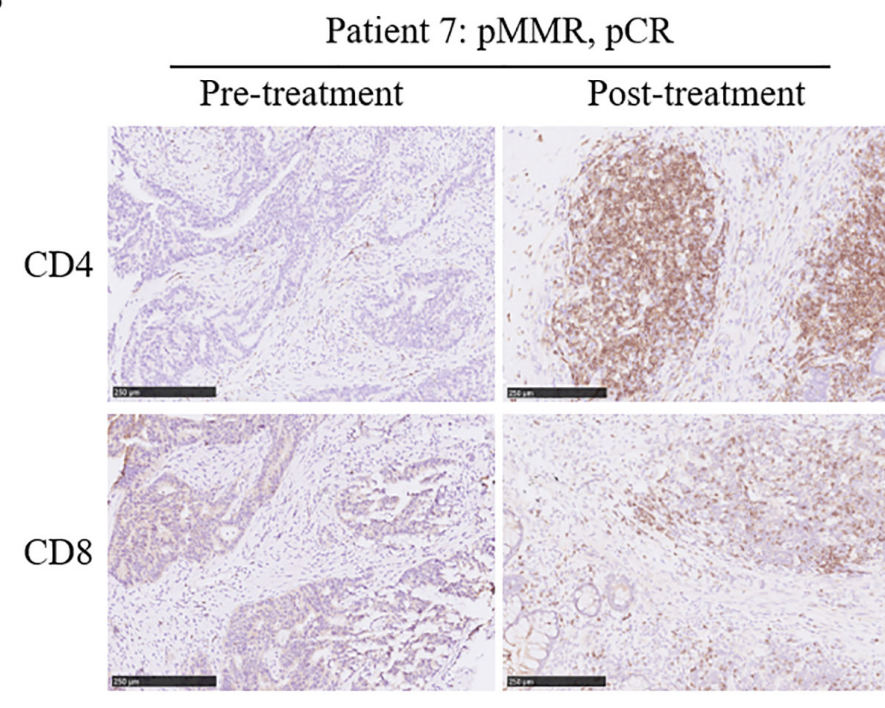

Post-treatment
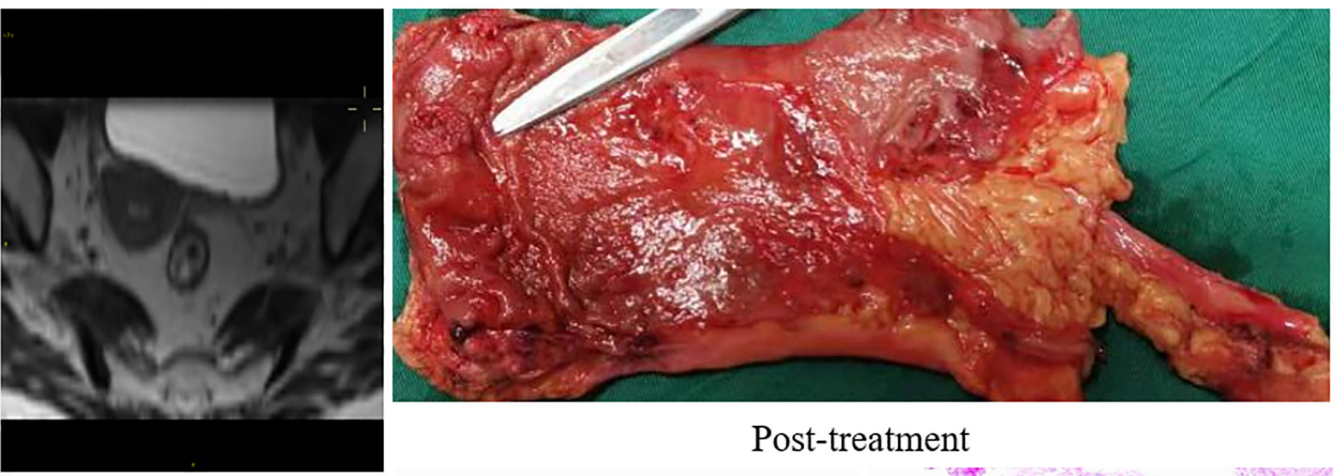

Post-treatment

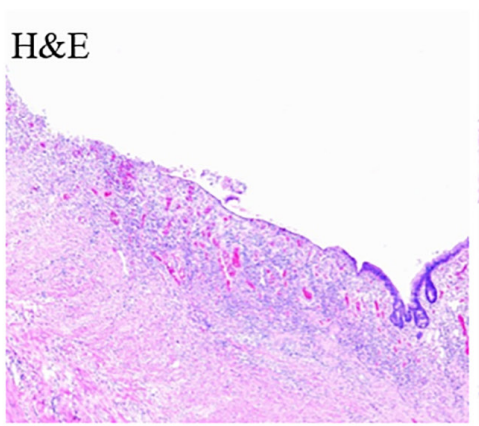

Figure 2 Pathological response to preoperative short-course radiotherapy followed by CAPOX and camrelizumab. (A) Radiological and pathological response from patient 7. Left: MRI of the abdomen (upper row) and endoscopic imaging of the primary tumor (lower row) before and after treatment. Right: Postoperative specimen with mucosal retraction at the tumor site with a polypoid structure (upper row) and H\&E staining showing CR of the primary tumor after treatment (lower row). (B) Representative CD8 and CD4 staining of pretreatment and posttreatment specimens of patient 7 and patient 12. Scale bars, $250 \mu \mathrm{m}$.

postoperative complications or treatment-related deaths occurred. The median interval from surgery to hospital discharge was 8 (range, 7-16) days.

The pCR (ypT0N0) rate was $48.1 \%(13 / 27)$, including $46.2 \%(12 / 26)$ for patients with pMMR and $100 \%(1 / 1)$ for those with dMMR. Negative nodes (ypN0) were reported in $19(70.4 \%)$ of the 27 patients, 18 of whom were in the pMMR patient subset $(69.2 \%, 18 / 26$; table 2$)$. As an example, imaging of patient 7 (a 63-year-old woman) with cT4N2bMx pMMR rectal cancer showed a visible tumor at baseline, the volume of which was significantly reduced after two cycles of treatment, and the histopathologic specimen revealed complete regression of the primary tumor, that is, pCR (figure 2A). Moreover, an obvious increase in $\mathrm{CD} 4^{+}$and $\mathrm{CD}^{+} \mathrm{T}$-cell infiltration was observed in posttreatment tumor samples compared with pretreatment samples in patient 7 but not in patient 12 who did not achieve pCR (figure 2B). Notably, all 
Table 3 Adverse events since the initiation of radiotherapy

\begin{tabular}{|c|c|c|}
\hline \multirow[b]{2}{*}{ Treatment-emergent AEs } & \multicolumn{2}{|c|}{ Patients $(n=30)$} \\
\hline & Any grade & Grade 3 \\
\hline Any $A E, n(\%)$ & $29(96.7)$ & $8(26.7)$ \\
\hline Leukopenia & $24(80.0)$ & $1(3.3)$ \\
\hline $\begin{array}{l}\text { Reactive cutaneous capillary } \\
\text { endothelial proliferation }\end{array}$ & $22(73.3)$ & 0 \\
\hline Radiation proctitis & $21(70.0)$ & 0 \\
\hline Anemia & $20(66.7)$ & $4(13.3)$ \\
\hline Peripheral neurotoxicity & $18(60.0)$ & 0 \\
\hline Neutropenia & $16(53.3)$ & $4(13.3)$ \\
\hline Thrombocytopenia & $16(53.3)$ & 0 \\
\hline Fatigue & $13(43.3)$ & 0 \\
\hline Anorexia & $10(33.3)$ & 0 \\
\hline Alanine transaminase increased & $9(30.0)$ & 0 \\
\hline Aspartate transaminase increased & $9(30.0)$ & 0 \\
\hline Vomiting & $8(26.7)$ & 0 \\
\hline Nausea & $6(20.0)$ & 0 \\
\hline Hypothyroidism & $2(6.7)$ & 0 \\
\hline Abdominal pain & $2(6.7)$ & 0 \\
\hline Hand-foot syndrome & $1(3.3)$ & 0 \\
\hline Constipation & $1(3.3)$ & 0 \\
\hline Diarrhea & $1(3.3)$ & 0 \\
\hline Immune-related AEs, n (\%)* & $22(81.5)$ & 0 \\
\hline $\begin{array}{l}\text { Reactive cutaneous capillary } \\
\text { endothelial proliferation }\end{array}$ & $22(81.5)$ & 0 \\
\hline Hypothyroidism & $2(7.4)$ & 0 \\
\hline
\end{tabular}

${ }^{*}$ The frequency of immune-related AEs among 27 patients who received at least one dose of camrelizumab was calculated. $\mathrm{AE}$, adverse event.

patients with PD-L1 CPS $\geq 1$ were reported to be ypN0. The pCR of patients with PD-L1 CPS $\geq 1$ was numerically higher than that of patients with CPS $<1(66.7 \%, 4 / 6$ vs $45.0 \%, 9 / 20, \mathrm{p}=0.2422)$.

\section{Safety}

Treatment-emergent AEs and immune-related AEs are summarized in table 3. At the data cut-off (January 15, 2021), the most common treatment-emergent AEs of any grade were leukopenia $(80.0 \%, 24 / 30)$, reactive cutaneous capillary endothelial proliferation $(73.3 \%$, $22 / 30)$, and radiation proctitis $(70.0 \%, 21 / 30)$. The most common grade 3 treatment-emergent AEs were neutropenia $(13.3 \%, 4 / 30)$ and anemia $(13.3 \%, 4 / 30)$. Immunerelated AEs were all grade 1-2, and the most common was reactive cutaneous capillary endothelial proliferation, which occurred in $22(81.5 \%)$ of 27 patients. Five patients experienced serious treatment-emergent AEs, among whom four were hospitalized or had prolonged hospitalization due to grade 2 radiation enteritis and one was hospitalized due to abdominal pain. No grade 4 or 5 $\mathrm{AE}$ occurred during the study.

\section{Results of biomarker analysis}

Next-generation sequencing was carried out in 53.8\% $(14 / 26)$ of patients with pMMR in this study; the remainder were not performed due to delivery of unqualified samples or patient refusal. As shown in figure 3A, the most frequently altered genes at baseline were TP53, $A P C$, and KRAS. Our small-sample analysis did not identify any genes with significant differences in the frequency of genomic alterations between the pCR and nonpCR groups (figure 3B). Compared with patients with TMB $<10$, those with TMB $\geq 10$ had a better tendency for pCR $(42.9 \%, 3 / 7$ vs $28.6 \%, 2 / 7, \mathrm{p}=0.3671)$. Interestingly, according to baseline copy number variant, none of the five patients with FGFR1-3 deletions achieved pCR, while more than half of the nine patients without FGFR1-3 deletions achieved, although no statistically significant difference was observed $(55.6 \%, 5 / 9$ vs $0 \%, 0 / 5, \mathrm{p}=0.086)$.

\section{DISCUSSION}

To the best of our knowledge, our study is the first to propose a new neoadjuvant therapy regimen of shortcourse hypofractionated radiotherapy combined with subsequent chemotherapy and anti-PD-1 antibody. In addition, this study provides preliminary evidence that the addition of camrelizumab to neoadjuvant SCRT followed by the CAPOX chemotherapy regimen results in a remarkable pCR for patients with LARC and is well tolerated, without new or unexpected safety issues.

As shown in previous studies, preoperative radiotherapy combined with chemotherapy resulted in tumor downstaging and reduced local recurrence, whereas pCR was observed only in $15 \%-30 \%$ of patients with rectal cancer. ${ }^{622}{ }^{24-26}$ In this study, our pCR rate of $48.1 \%$ is encouraging, meaning that our innovative preoperative combination therapy strategy provides more opportunities for sphincter-preserving surgery and also raises the prospect that more patients with LARC, especially those with low rectal cancer, may achieve a clinical complete response and have a watch-and-wait strategy of nonsurgical treatment implemented to improve their quality of life.

Immunotherapy is generally ineffective in the pMMR/ MSS tumors that constitute the majority of CRCs, which could be attributed to insufficient lymphocytic infiltration. ${ }^{727}$ Preclinical data have shown that radiotherapy can sensitize refractory tumors to PD-1/PD-L1 blockade by modulating the immunogenicity of tumor cells, enhancing antigen-specific $\mathrm{CD}^{+}{ }^{+} \mathrm{T}$-cell responses, and increasing PD-L1 expression on tumor cells and immune cells in the tumor microenvironment ${ }^{1628}$; in addition, chemotherapy can also upregulate PD-L1 on dendritic cells and increase immune-cell infiltration. ${ }^{29}{ }^{30}$ Based on these rationales, immunotherapy strategies combined with chemoradiotherapy are being explored in patients 
A

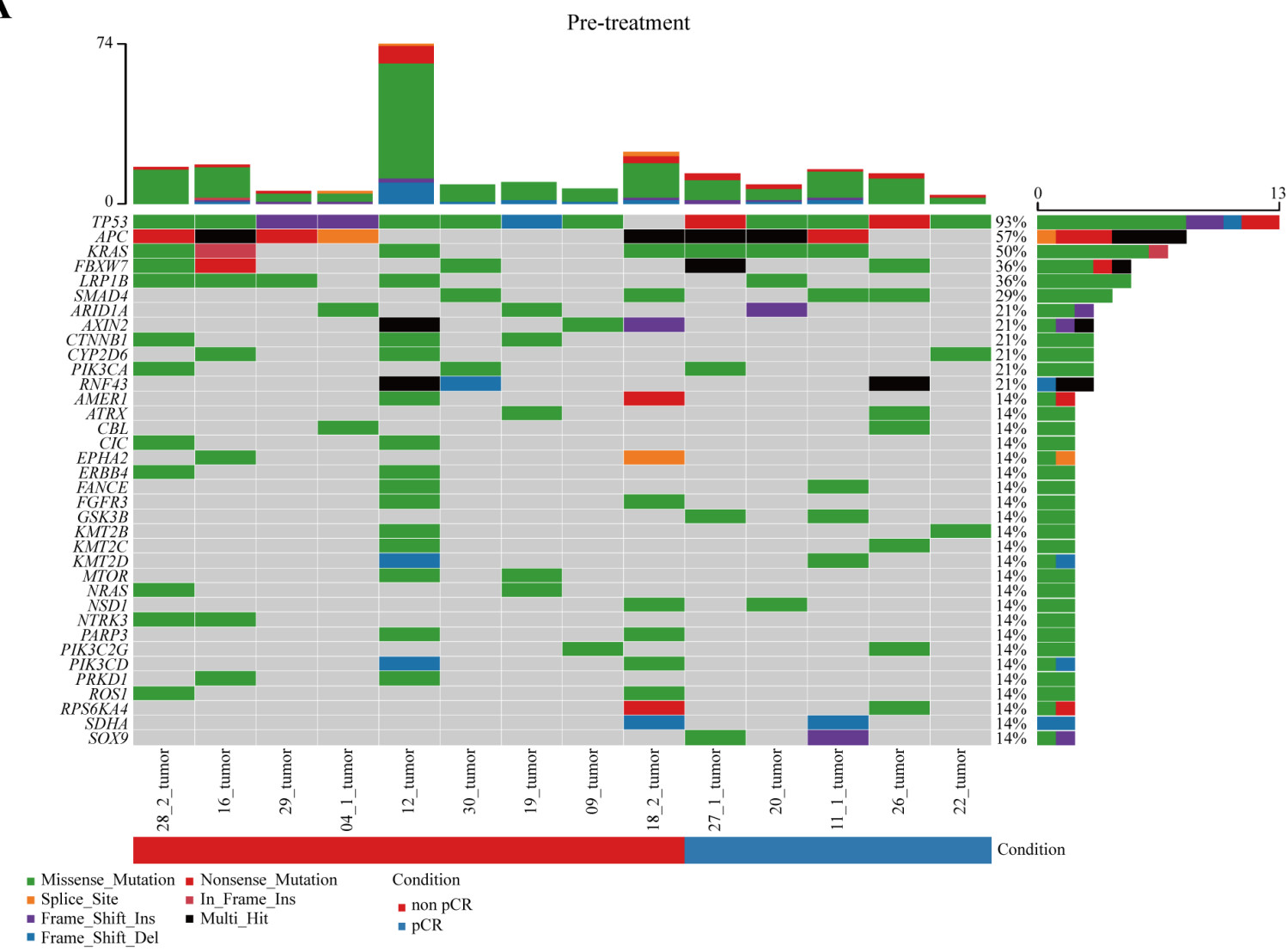

B

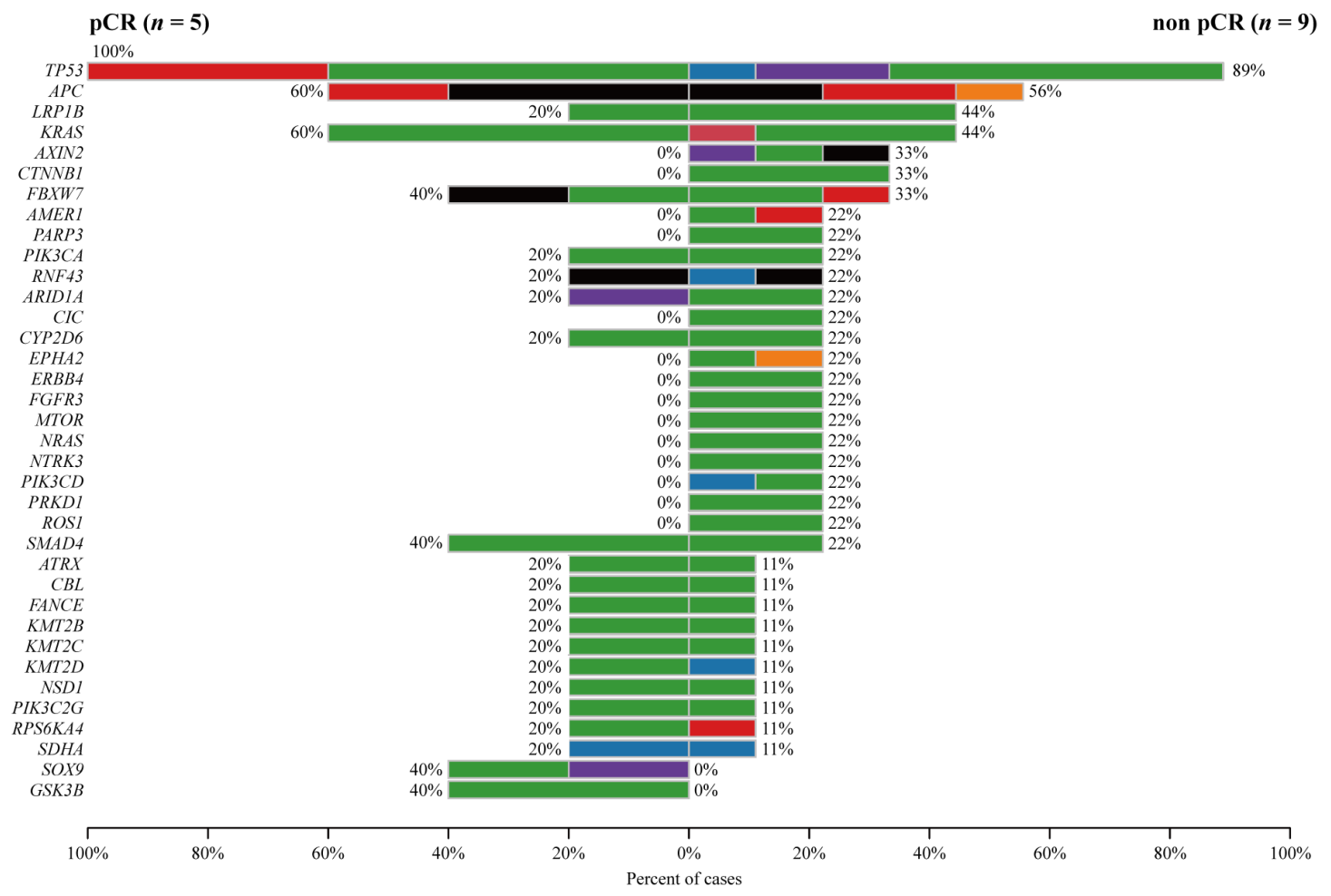

- Frame_Shift_Del a Nonsense_Mutation

- Frame Shift_Ins In Frame Ins

- Missense_Mutation $\quad$ - Splice_Site

- Multi Hit

Figure 3 Genetic analysis. (A) Overall frequency of gene alterations at baseline. (B) Frequency of genomic alterations between the $\mathrm{pCR}$ and non-pCR groups. pCR, pathological complete response. 
with pMMR/MSS rectal cancer, especially in LARC setting. In the VOLTAGE study, the pCR rate was $30 \%$ in patients with MSS LARC receiving preoperative LC-CRT and sequential nivolumab. ${ }^{17}$ In our study, the pCR rate was $46.2 \%$ for patients with pMMR disease. In addition, the recently reported pCR rate was $37.5 \%$ among patients with locally advanced rectal adenocarcinoma receiving SCRT followed by mFOLFOX-6 plus avelumab (an antiPD-L1 antibody) as neoadjuvant therapy in the Averectal study. ${ }^{31}$ When comparing the results of our study and the Averectal study, differential $\mathrm{N}$ staging was noted between the enrolled patients, with stage N1 patients being predominant in our study $(53.3 \%)$ but stage N2 in the Averectal study (75.0\%). ${ }^{31}$ In addition, a meta-analysis of randomized trials has indicated the superior efficacy of anti-PD-1 antibody over anti-PD-L1 antibody in solid tumors, regardless of monotherapy or combination strategies. ${ }^{32}$ Although the course of neoadjuvant immunotherapy plus chemotherapy is inconclusive, a short course of chemotherapy has been shown to improve the early efficacy of immunotherapy, as reported by Checkmate 9LA. ${ }^{33}$ Results from the NRG-GI002 study demonstrated neoadjuvant concurrent pembrolizumab and LC-CRT after FOLFOX induction did not significantly improve the short-term clinical outcomes of patients with LARC compared with FOLFOX and LC-CRT alone, with pCR rate of $31.9 \%$ in pembrolizumab group versus $29.4 \%$ in control group $(\mathrm{p}=0.75) .{ }^{34}$ Although cross-study comparisons should be made with caution, it is interesting that that the pCR rate of $48.1 \%$ observed in our study compared favorably with the NRG-GI002 study. The differences may be explained by different neoadjuvant therapeutic strategies with the differential timing of anti-PD-1 antibody administration.

A time interval of at least 7 days after SCRT has been reported to be probably required to achieve a favorable immune response, suggesting that the choice of the timing of immunotherapy after SCRT is important. ${ }^{35}$ The PACIFIC trial reported that the initiation of durvalumab within 2 weeks after chemoradiotherapy (rather than $\geq 2$ weeks) was linked to a higher clinical benefit. ${ }^{36}$ Therefore, we have reasons to believe that our choice of timing for the initiation of immune checkpoint inhibitor camrelizumab in this study (median 12 days) is appropriate.

The previous VOLTAGE-A study showed that positive PD-L1 expression and high TMB are good predictors of neoadjuvant efficacy from LC-CRT followed by nivolumab. ${ }^{18}$ Similarly, a trend toward a better pCR rate was observed in patients with PD-L1 CPS $\geq 1$ or with $\mathrm{TMB} \geq 10$ in our phase II trial, although the differences were not statistically significant. Given that ours was an exploratory analysis with a small sample, we recommend that this finding be interpreted with caution. In addition, patients without FGFR1-3 deletions appear to be more likely to achieve clinical benefit from our neoadjuvant strategy than those with FGFR1-3 deletions (55.6\% vs $0 \%)$. In urothelial carcinoma, patients with FGFR mutations have been reported to show a worse response to immunotherapy than those without FGFR mutations. ${ }^{37}$ A similar result was demonstrated in metastatic gastric adenocarcinoma, showing that the patient harboring MSI-H and FGFR2 alterations was resistant to anti-PD-1 monotherapy. ${ }^{38}$ If positively validated in the subsequent larger study that we plan to conduct, the presence of FGFR1-3 deletions may be a potent predictor of response to neoadjuvant immunotherapy in rectal cancer.

The limitations of this study consist of the small sample size of patients, lack of a control group, and insufficient postoperative follow-up time. Furthermore, whether improvement in pCR in our study can contribute to better survival needs further exploration. A large multicenter randomized phase III study is going to confirm the value of preoperative short-course hypofractionated radiotherapy combined with subsequent chemotherapy and camrelizumab regimen (NCT04928807).

In conclusion, SCRT combined with subsequent CAPOX plus camrelizumab followed by delayed surgery showed a favorable pCR rate with manageable toxicities, especially in the pMMR setting. This suggests that neoadjuvant SCRT combined with subsequent CAPOX plus camrelizumab is a promising feasible strategy and is expected to provide more opportunities for surgery to be delayed or avoided among patients with middle-low LARC and for organ function to be preserved in the future.

\section{Author affiliations \\ ${ }^{1}$ Cancer Center, Union Hospital, Tongji Medical College, Huazhong University of Science and Technology, Wuhan, China \\ ${ }^{2}$ Department of Gastrointestinal Surgery, Union Hospital, Tongji Medical College, Huazhong University of Science and Technology, Wuhan, China \\ ${ }^{3}$ Department of Digestive Surgical Oncology, Union Hospital, Tongji Medical College, Huazhong University of Science and Technology, Wuhan, China \\ ${ }^{4}$ Department of Radiology, Union Hospital, Tongji Medical College, Huazhong University of Science and Technology, Wuhan, China \\ ${ }^{5}$ Department of Pathology, Union Hospital, Tongji Medical College, Huazhong University of Science and Technology, Wuhan, China \\ ${ }^{6}$ Department of Medical Affairs, Jiangsu Hengrui Pharmaceuticals Co., Ltd, Shanghai, China}

Correction notice An error in Figure 2A has been corrected since this article was first published. The left image of H\&E staining should have been labelled as posttreatment.

Acknowledgements We thank the patients and their families as well as all other participants who made the study possible. We thank Haihong Wang for her contribution to the collection and management of specimens, Yali Kong (a former employee of Jiangsu Hengrui Pharmaceuticals Co., Ltd.) for her input in data analysis, and Xinzhi Gao (Jiangsu Hengrui Pharmaceuticals Co., Ltd.) for her careful revision of the manuscript. We also thank Yanhua Xu (a medical writer at Jiangsu Hengrui Pharmaceuticals Co., Ltd.) for the medical writing assistance in accordance with Good Publication Practice guidelines.

Contributors $\mathrm{KT}, \mathrm{TZ}, \mathrm{ZH}$, and $\mathrm{CM}$ conceived and designed this study. ZL, MC, PZ, $\mathrm{GL}, \mathrm{TL}, \mathrm{XL}, \mathrm{KC}, \mathrm{JW}, \mathrm{JL}, \mathrm{HL}, \mathrm{WZ}, \mathrm{JG}, \mathrm{CW}, \mathrm{LW}, \mathrm{JF}$, LZ, and ZW enrolled patients and collected the data. TZ, ZL, MC, PZ, and XN participated in the data curation. XL, XN, $\mathrm{JF}$, and $\mathrm{LZ}$ analyzed the data, and all authors participated in data interpretation. $\mathrm{XL}$, $\mathrm{JF}, \mathrm{KY}$, and GW provided the administrative support. KT, ZL, MC, and PZ drafted the manuscript and all authors reviewed. KT and TZ had full access to all the data in the study and had final responsibility for the decision to submit for publication. The final version was approved to be submitted by all authors. KT and TZ are guarantors of the work. 
Funding This work was supported by Ministry of Science and Technology of China (2018YFC1313302), 2018 National Natural Science Foundation of China (81874061), and Jiangsu Hengrui Pharmaceuticals Co., Ltd. (no grant number).

Competing interests TZ received research funding from Jiangsu Hengrui Pharmaceuticals Co., Ltd. ZH and CM are employees of Jiangsu Hengrui Pharmaceuticals Co., Ltd. All other authors declare no potential conflicts of interest.

Patient consent for publication Not applicable.

Ethics approval The study was approved by the ethics committee of Union Hospital affiliated to Tongji Medical College of Huazhong University of Science and Technology, and conducted in accordance with the Declaration of Helsinki and Good Clinical Practice guidelines. All patients provided written informed consent.

Provenance and peer review Not commissioned; externally peer reviewed.

Data availability statement Data used and analyzed during this study are available from the corresponding author on reasonable request.

Supplemental material This content has been supplied by the author(s). It has not been vetted by BMJ Publishing Group Limited (BMJ) and may not have been peer-reviewed. Any opinions or recommendations discussed are solely those of the author(s) and are not endorsed by BMJ. BMJ disclaims all liability and responsibility arising from any reliance placed on the content. Where the content includes any translated material, BMJ does not warrant the accuracy and reliability of the translations (including but not limited to local regulations, clinical guidelines, terminology, drug names and drug dosages), and is not responsible for any error and/or omissions arising from translation and adaptation or otherwise.

Open access This is an open access article distributed in accordance with the Creative Commons Attribution Non Commercial (CC BY-NC 4.0) license, which permits others to distribute, remix, adapt, build upon this work non-commercially, and license their derivative works on different terms, provided the original work is properly cited, appropriate credit is given, any changes made indicated, and the use is non-commercial. See http://creativecommons.org/licenses/by-nc/4.0/.

\section{ORCID iD}

Tao Zhang http://orcid.org/0000-0003-4018-3393

\section{REFERENCES}

1 International Agency for Research on Cancer. Global cancer observatory: cancer tomorrow, 2021. Available: https://gco.iarc.fr/ tomorrow/ [Accessed 1 Feb 2021].

2 Feng R-M, Zong Y-N, Cao S-M, et al. Current cancer situation in China: good or bad news from the 2018 Global Cancer Statistics? Cancer Commun 2019;39:22.

3 Erlandsson J, Holm T, Pettersson D, et al. Optimal fractionation of preoperative radiotherapy and timing to surgery for rectal cancer (Stockholm III): a multicentre, randomised, non-blinded, phase 3, non-inferiority trial. Lancet Oncol 2017;18:336-46.

4 Erlandsson J, Lörinc E, Ahlberg M, et al. Tumour regression after radiotherapy for rectal cancer - results from the randomised Stockholm III trial. Radiother Oncol 2019;135:178-86.

5 Jin J, Liu S, Zhu Y. The updated results for the phase 3 study of $5 \times 5$ Gy followed by chemotherapy in locally advanced rectal cancer (stellar trial). Int J Radiat Oncol Biol Phys 2017;99:E157.

6 Bahadoer RR, Dijkstra EA, van Etten B, et al. Short-course radiotherapy followed by chemotherapy before total mesorectal excision (TME) versus preoperative chemoradiotherapy, TME, and optional adjuvant chemotherapy in locally advanced rectal cancer (RAPIDO): a randomised, open-label, phase 3 trial. Lancet Oncol 2021;22:29-42.

7 Le DT, Uram JN, Wang H, et al. PD-1 blockade in tumors with mismatch-repair deficiency. N Engl J Med 2015;372:2509-20.

8 Luchini C, Bibeau F, Ligtenberg MJL, et al. ESMO recommendations on microsatellite instability testing for immunotherapy in cancer, and its relationship with PD-1/PD-L1 expression and tumour mutational burden: a systematic review-based approach. Ann Oncol 2019;30:1232-43.

9 Ganesh K, Stadler ZK, Cercek A, et al. Immunotherapy in colorectal cancer: rationale, challenges and potential. Nat Rev Gastroenterol Hepatol 2019;16:361-75.

10 Wang D, Yu W, Lian J, et al. Th17 cells inhibit CD8 ${ }^{+} \mathrm{T}$ cell migration by systematically downregulating CXCR3 expression via IL-17A/ STAT3 in advanced-stage colorectal cancer patients. J Hematol Oncol 2020;13:68.
11 Tumeh PC, Harview CL, Yearley JH, et al. PD-1 blockade induces responses by inhibiting adaptive immune resistance. Nature 2014;515:568-71.

12 Cohen R, Shi Q, André T. Immunotherapy for early stage colorectal cancer: a glance into the future. Cancers 2020;12 doi:10.3390/ cancers 12071990

13 Chalabi M, Fanchi LF, Dijkstra KK, et al. Neoadjuvant immunotherapy leads to pathological responses in MMR-proficient and MMRdeficient early-stage colon cancers. Nat Med 2020;26:566-76.

14 Gong J, Le TQ, Massarelli E, et al. Radiation therapy and PD-1/ PD-L1 blockade: the clinical development of an evolving anticancer combination. J Immunother Cancer 2018;6:46.

15 Antonia SJ, Villegas A, Daniel D, et al. Durvalumab after chemoradiotherapy in stage III non-small-cell lung cancer. $N$ Engl J Med 2017;377:1919-29.

16 Sharabi AB, Lim M, DeWeese TL, et al. Radiation and checkpoint blockade immunotherapy: radiosensitisation and potential mechanisms of synergy. Lancet Oncol 2015;16:e498-509.

17 et alYuki S, Bando H, Tsukada Y. Short-term results of VOLTAGE-A: nivolumab monotherapy and subsequent radical surgery following preoperative chemoradiotherapy in patients with microsatellite stable and microsatellite instability-high locally advanced rectal cancer. Presented at: 2020 ASCO Virtual Scientific Program. Abstract 4100. Available: https://meetinglibrary.asco.org/record/ 188255/abstract

18 et allnamori K, Togashi Y, Bando $\mathrm{H}$. Translational research of voltage-A1: efficacy predictors of preoperative chemoradiotherapy and subsequent nivolumab monotherapy in patients with microsatellite-stable locally advanced rectal cancer. Presented at: 2020 ASCO Virtual Scientific Program. Abstract 4073. Available: https://meetinglibrary.asco.org/record/187897/abstract

19 Nesseler JP, Lee M-H, Nguyen C, et al. Tumor size mattersunderstanding concomitant tumor immunity in the context of hypofractionated radiotherapy with immunotherapy. Cancers $2020 ; 12$ doi:10.3390/cancers12030714

20 Quirke P, Durdey P, Dixon MF, et al. Local recurrence of rectal adenocarcinoma due to inadequate surgical resection. Histopathological study of lateral tumour spread and surgical excision. Lancet 1986;2:996-9.

21 Ryan R, Gibbons D, Hyland JMP, et al. Pathological response following long-course neoadjuvant chemoradiotherapy for locally advanced rectal cancer. Histopathology 2005;47:141-6.

22 Bujko K, Wyrwicz L, Rutkowski A, et al. Long-course oxaliplatinbased preoperative chemoradiation versus $5 \times 5$ Gy and consolidation chemotherapy for cT4 or fixed cT3 rectal cancer: results of a randomized phase III study. Ann Oncol 2016;27:834-42.

23 Rödel C, Liersch T, Becker H, et al. Preoperative chemoradiotherapy and postoperative chemotherapy with fluorouracil and oxaliplatin versus fluorouracil alone in locally advanced rectal cancer: initial results of the German CAO/ARO/AIO-04 randomised phase 3 trial. Lancet Oncol 2012;13:679-87.

24 Fokas E, Allgäuer M, Polat B, et al. Randomized phase II trial of chemoradiotherapy plus induction or consolidation chemotherapy as total neoadjuvant therapy for locally advanced rectal cancer: CAO/ ARO/AIO-12. J Clin Oncol 2019;37:3212-22.

25 Deng Y, Chi P, Lan P, et al. Modified FOLFOX6 with or without radiation versus fluorouracil and leucovorin with radiation in neoadjuvant treatment of locally advanced rectal cancer: initial results of the Chinese FOWARC multicenter, open-label, randomized three-arm phase III trial. J Clin Oncol 2016;34:3300-7.

26 Zhu J, Liu A, Sun X, et al. Multicenter, randomized, phase III trial of neoadjuvant chemoradiation with capecitabine and irinotecan guided by UGT1A1 status in patients with locally advanced rectal cancer. $J$ Clin Oncol 2020;38:4231-9.

27 Eng C, Kim TW, Bendell J, et al. Atezolizumab with or without cobimetinib versus regorafenib in previously treated metastatic colorectal cancer (IMblaze370): a multicentre, open-label, phase 3, randomised, controlled trial. Lancet Oncol 2019;20:849-61.

28 Spiotto M, Fu Y-X, Weichselbaum RR. The intersection of radiotherapy and immunotherapy: mechanisms and clinical implications. Sci Immunol 2016;1:EAAG1266.

29 Heinhuis KM, Ros W, Kok M, et al. Enhancing antitumor response by combining immune checkpoint inhibitors with chemotherapy in solid tumors. Ann Oncol 2019;30:219-35.

30 Tesniere A, Schlemmer F, Boige V, et al. Immunogenic death of colon cancer cells treated with oxaliplatin. Oncogene 2010;29:482-91.

31 Shamseddine A, Zeidan Y, Bouferraa Y. Efficacy and safety of neoadjuvant short-course radiation followed by mFOLFOX-6 plus avelumab for locally-advanced rectal adenocarcinoma: Averectal study. Presented at: ESMO World GI 2021, 2021. https://www.hmpg loballearningnetwork.com/node/206762 
32 Duan J, Cui L, Zhao X, et al. Use of immunotherapy with programmed cell death 1 vs programmed cell death ligand 1 inhibitors in patients with cancer: a systematic review and metaanalysis. JAMA Oncol 2020;6:375-84.

33 Paz-Ares L, Ciuleanu T-E, Cobo M, et al. First-line nivolumab plus ipilimumab combined with two cycles of chemotherapy in patients with non-small-cell lung cancer (CheckMate 9LA): an international, randomised, open-label, phase 3 trial. Lancet Oncol 2021;22:198-211.

34 Rahma OE, Yothers G, Hong TS, et al. Use of total neoadjuvant therapy for locally advanced rectal cancer: initial results from the pembrolizumab arm of a phase 2 randomized clinical trial. JAMA Oncol 2021;7:1225-30.
35 Glynne-Jones R, Hall M, Nagtegaal ID. The optimal timing for the interval to surgery after short course preoperative radiotherapy $(5 \times 5$ Gy) in rectal cancer - are we too eager for surgery? Cancer Treat Rev 2020;90:102104.

36 Faivre-Finn C, Spigel DR, Senan S, et al. Efficacy and safety evaluation based on time from completion of radiotherapy to randomization with durvalumab or placebo in pts from PACIFIC. Annals of Oncology 2018;29:viii488.

37 Loriot Y, Necchi A, Park SH, et al. Erdafitinib in locally advanced or metastatic urothelial carcinoma. N Engl J Med 2019;381:338-48.

38 Kwon M, An M, Klempner SJ, et al. Determinants of response and intrinsic resistance to PD-1 blockade in microsatellite instability-high gastric cancer. Cancer Discov 2021;11:2168-85. 


\section{Correction: Phase II, single-arm trial of preoperative short- course radiotherapy followed by chemotherapy and camrelizumab in locally advanced rectal cancer}

Lin Z, Cai M, Zhang P, et al. Phase II, single-arm trial of preoperative short-course radiotherapy followed by chemotherapy and camrelizumab in locally advanced rectal cancer. J ImmunoTher Cancer 2021;9:e003554. doi:10.1136/jitc-2021-003554

This article has been corrected since it was first published. There was an error in panel A of Figure 2; the left image of H\&E staining is mistakenly described as pre-treatment, while in fact it is a post-treatment image. The figure has now been updated.

Open access This is an open access article distributed in accordance with the Creative Commons Attribution Non Commercial (CC BY-NC 4.0) license, which permits others to distribute, remix, adapt, build upon this work non-commercially, and license their derivative works on different terms, provided the original work is properly cited, appropriate credit is given, any changes made indicated, and the use is non-commercial. See http://creativecommons.org/licenses/by-nc/4.0/.

(c) Author(s) (or their employer(s)) 2022. Re-use permitted under CC BY-NC. No commercial re-use. See rights and permissions. Published by BMJ.

J Immunother Cancer 2022;10:e003554corr1. doi:10.1136/jitc-2021-003554corr1

A) Check for updates 